\title{
THE NACHBIN COMPACTIFICATION VIA CONVERGENCE ORDERED SPACES
}

\author{
D.C. KENT and DONGMEI LIU \\ Department of Mathematics \\ Washington State University \\ Pullman, Washington 99164-3113
}

(Received April 21, 1992)

\begin{abstract}
We construct the Nachbin compactification for a $T_{3.5}$-ordered topological ordered spdice by taking a quotient of an ordered convergence space compactification. A variation of this quotient construction leads to a compactification functor on the category of $T_{3.5}$-ordered convergence ordered spaces.
\end{abstract}

KEY WORDS AND PHRASES: topological ordered space, convergence ordered space, $T_{2}$-ordered space, $T_{3.5}$-ordered space, Nachbin compactification.

1980 MATHEMATICS SUBJECT CLASSIFICATION CODE: 54 D 35, 54 F 05, 54 A 20

\section{INTRODUCTION.}

The Nachbin (or Stone-Cech-ordered) compactification (see [1], [6]) is the largest $T_{2}$-ordered topological ordered compactification of a $T_{3.5}$-ordered topological ordered space. In [4], one of the authors and G.D. Richardson constructed an ordered compactification $\left(X^{*}, \varphi\right)$ for an arbitrary convergence ordered space $X$. This latter compactification exhibits essentially the same universal property as the Nachbin compactification, but behaves poorly relative to separation properties (see Example 1.4).

Starting with an arbitrary convergence ordered space $X$, we introduce an equivalence relation $R$ on the set $\left|X^{*}\right|$ which underlies $X^{*}$, and obtain an ordered quotient space $X^{*} / R$ which is both compact and $T_{2}$-ordered. We next give two conditions $C$ and $O$ which are necessary and sufficient to make the natural map from $X$ into $X * / R$ both an order embedding and a homeomorphic embedding, so that $X^{*} / R$ becomes a $T_{2}$-ordered convergence ordered compactification of $X$. For ordered convergence spaces $X$ satisfying conditions $C$ and $O$, it turns out that the topological modification $\lambda X$ of $X$ is a $T_{3.5}$-ordered topological ordered space, and $\lambda\left(X^{*} / R\right)$ is the Nachbin compactification of $\lambda X$. In particular, if $X$ is assumed to be a $T_{3.5}$-ordered topological ordered space, then $\lambda\left(X^{*} / R\right)$ is the Nachbin compactification of $\boldsymbol{X}$.

In addition to giving an alternate construction for the Nachbin compactification, we obtain some interesting results pertaining to convergence ordered compactifications. In Section 3, we define a 
regular convergence ordered space satisfying conditions $C$ and $O$ to be a $T_{3.5}$-ordered convergence ordered space, and we show that for such a space $X$, the regular modification $r\left(X^{*} / R\right)$ of the quotient $X \bullet / R$ is a regular, $T_{2}$-ordered convergence ordered compactification of $X$. Relative to this compactification functor, the regular, $T_{2}$-ordered, compact convergence spaces (with increasing, continuous maps as morphisms) form an epireflective subcategory of the category of all $T_{3.5}$-ordered convergence ordered spaces (with increasing, continuous maps as morphisms).

\section{PRELIMINARIES.}

We introduce some basic notation and terminology and summarize some results from [4]. If $(X, \leq)$ is a poset, and $A \subseteq X$, we denote by $i(A), d(A)$, and $A^{\wedge}$ the increasing, decreasing, and convex hulls, respectively, of $A$; note that $A^{\wedge}=i(A) \cap d(A)$. Similarly, if $F(X)$ is the set of all (proper) filters on $X$ and $\mathcal{F} \in \boldsymbol{F}(\mathrm{X})$, let $i(\mathcal{F})$, the filter generated by $\{i(F): F \in \mathcal{F}\}$, be the increasing hull of $\mathcal{F}$; the decreasing hull $d(\xi)$ and convex hull $f$ are defined analagously. A filter $₹$ is said to be convex if $\mathcal{F}=\hat{\xi}$. Note that $\hat{\xi}=i(\mathcal{F}) \vee d(\mathcal{F})$.

If $(X, \leq, \rightarrow)$ is a poset $(X, \leq)$ equipped with a convergence structure $\rightarrow$ which is locally convex (i.e., $\hat{f} \rightarrow x$ whenever $\mathcal{F} \rightarrow x$ ), then $(X, \leq, \rightarrow)$ is called a convergence ordered space; we usually write $X$ rather than $(X, \leq, \rightarrow)$ when there is no danger of ambiguity. A convergence ordered space is $T_{1}$-ordered if the sets $i(x)$ and $d(x)$ are closed for all $x \in X$, and $T_{2}$-ordered if the order $\leq$ is a closed subset of $X \times X$. For any convergence ordered space $X$, let $C I^{*}(X)$ (respectively, $C D^{*}(X)$ ) denote the set of all continuous, increasing (respectively, decreasing) maps from $X$ into $[0,1]$.

A convergence ordered space whose convergence structure is a topology is called a topological ordered space. Such a space is said to be convex if the open monotone (i.e., increasing or decreasing) sets form a subbase for the topology. For the remainder of this paper, we shall adopt the notational abbreviation used in [4] and write "t.o.s" instead of "topological ordered space" and "c.o.s." in place of "convergence ordered space".

A t.o.s. $X$ is said to be $T_{3.5}$-ordered if it satisfies the following conditions: (1) If $x \in X, A$ is a closed subset of $X$, and $x \notin A$, then there is $f \in C I^{\circ}(X)$ and $g \in C D^{*}(X)$ such that $f(x)=g(x)=0$ and $f(y) \vee g(y)=1$, for all $y \in A$; (2) If $x \not y$ in $X$, there is $f \in C I^{*}(X)$ such that $f(y)=0$ and $f(x)=1$. The $T_{3.5}$-ordered spaces are precisely those which allow $T_{2}$-ordered t.o.s. compactifications, and all $T_{3.5}$-ordered spaces are convex.

If $X$ is a $T_{3.5}$-ordered t.o.s., then the Nachbin compactification of $X$ (see [1], [6]) is obtained by embedding $X$ into an "ordered cube", whose component intervals are indexed by $C I^{*}(X)$. The Nachbin compactification $\beta_{0} X$ is characterized by the following well-known result.

PROPOSITION 1.1. If $X$ is a $T_{3.5}$-ordered t.o.s., then $\beta_{0} X$ is $T_{2}$-ordered. Furthermore, if $f: X \rightarrow Y$ is an increasing, continuous map and $Y$ is a compact, $T_{2}$-ordered t.o.s., then $f$ has a unique, increasing, continuous extension $f^{\prime}: \beta_{0} X \rightarrow Y$.

We next describe briefly the construction of the convergence ordered compactification $X^{*}$ of an arbitrary c.o.s. $X$ described in [4], which has essentially the same lifting property as $\beta_{0} X$. Given a c.o.s. $X$, let $X^{\prime}$ be the set of all non-convergent maximal convex filters on $X$, and let $X^{*}=\{\dot{x}: x \in X\} \cup X^{\prime}$. Before proceeding further, it will be useful to establish the following proposition about maximal convex filters.

PROPOSITION 1.2. The maximal convex filters on a poset $X$ are precisely the set $\{\hat{f}: \mathcal{F}$ is an ultrafilter on $X$ \}.

PROOF. Clearly every maximal convex filter is the convex hull of every finer ultrafilter. Conversely, suppose $\mathcal{F}$ is an ultrafilter on $X$ and $\mathcal{G}$ is a convex filter such that $\hat{f} \leq \mathcal{G}$. Then for any 
convex set $G \in \mathcal{G}$, the filters $\mathcal{F}_{1}$ and $\mathcal{F}_{2}$ generated by $\{i(G) \cap F: F \in \mathcal{F}\}$ and $\{d(G) \cap F: F \in \mathcal{F}\}$, respectively, are well-defined filters finer than, and hence equal to, $\mathcal{F}$. Thus $i(G) \in \mathcal{F}$ and $d(G) \in \mathcal{F}$ implies $i(G) \cap d(G)=G \in \mathcal{F}$; therefore $\mathcal{G}=\hat{\mathcal{F}}$.

Again assuming that $X$ is an arbitrary c.o.s., let $\varphi: X \rightarrow X^{\bullet}$ be defined by $\varphi(x)=\dot{x}$, for all $x \in X$. A partial order $\leq^{\bullet}$ is defined on $X^{\bullet}$ as follows: $\mathcal{F} \leq^{\bullet} \mathcal{G}$ iff $i(\mathcal{F}) \leq \mathcal{G}$ (or, equivalently, $d(\mathcal{G}) \leq \mathcal{F})$. Since $x \leq y$ iff $\dot{x} \leq^{*} \dot{y}, \varphi:(X, \leq) \rightarrow\left(X^{*}, \leq^{\bullet}\right)$ is an order embedding.

If $A \subseteq X$, let $A^{\bullet}=\left\{\mathcal{F} \in X^{\bullet}: A \in \mathcal{F}\right\}$; if $\mathcal{F} \in \boldsymbol{F}(X)$, let $\mathcal{F}^{*}$ denote the filter in $F\left(X^{\bullet}\right)$ generated by $\left\{F^{\bullet}: F \in \mathcal{F}\right\}$. A convergence structure $\stackrel{*}{\rightarrow}$ on $\left(X^{*}, \leq^{*}\right)$ is defined as follows: For $A \in F\left(X^{*}\right)$,

$$
\begin{aligned}
& A \stackrel{*}{\rightarrow} \dot{x} \in \varphi(X) \text { iff there is } \mathcal{F} \rightarrow x \text { such that } \mathcal{F}^{*} \leq \mathcal{A} \text {; } \\
& A \stackrel{*}{\rightarrow} \mathcal{G} \in X^{\prime} \text { iff } \mathcal{G}^{*} \leq A \text {. }
\end{aligned}
$$

Writing $X^{*}$ in place of $\left(X^{*}, \leq^{*}, \stackrel{*}{\rightarrow}\right)$, we state the following result which is proved in [4].

PROPOSITION 1.3. If $X$ is a c.o.s., then $\left(X^{*}, \varphi\right)$ is a convergence ordered compactification of $X$. If $f: X \rightarrow Y$ is a continuous, increasing map and $Y$ a compact, regular, $T_{2}$-ordered c.o.s., then $f$ has a unique, increasing, continuous extension $f_{*}: X^{*} \rightarrow Y$.

Recall that a convergence space $Y$ is regular if $c l_{Y} F \rightarrow x$ whenever $\mathcal{F} \rightarrow x$. Here "cly" is the closure operator for $Y$, and $c l_{Y} F$ is the filter on $Y$ generated by $\left\{c l_{Y} F: F \in \mathcal{F}\right\}$.

In [4], a c.o.s. $X$ is defined to be strongly $T_{2}$-ordered if $X$ is $T_{2}$ (i.e., convergent filters have unique limits) and the following conditions hold: $\left(S_{1}\right)$ if $\mathcal{F} \rightarrow x, \mathcal{G} \in X^{\prime}$, and $i(\mathcal{F}) \leq \mathcal{G}$, then $d(\mathcal{G}) \leq \dot{x}$; $\left(S_{2}\right)$ if $\mathcal{F} \rightarrow x, \mathcal{G} \in X^{\prime}$, and $d(\mathcal{F}) \leq \mathcal{G}$, then $i(\mathcal{G}) \leq \dot{x}$. In Proposition 2.8, [4], it is shown that $X^{*}$ is $T_{2}$-ordered iff $X$ is strongly $T_{2}$-ordered. As we see in the next example, very nice c.o.s.'s may fail to be strongly $T_{2}$-ordered.

EXAMPLE 1.4. Let $X$ be the Euclidean plane with its usual (product) order and topology. Let $\xi$ be the filter on $X$ generated by sets of the form $F_{n}=\left\{(a, b) \in X:-\frac{1}{n}<a<0, \quad b=0\right\}$ for each natural number $n$, and let $x=(0,0)$. Let $\mathcal{G}$ be the convex hull of any ultrafilter containing the set $S=\left\{(a, b) \in X: a=-b^{-1}\right\}$ and coarser than the filter $\mathcal{H}$ generated by sets of the form $H_{n}=\{(a, b) \in X: b \geq n\}$ for $n=1,2,3, \ldots$. Note that $\left(S_{1}\right)$ is violated by $\mathcal{F}, \mathcal{G}$ and $x$; thus the compactification $X^{*}$ of $X$ is not $T_{2}$-ordered.

\section{2. $\beta_{0} X$ AS A QUOTIENT OF $X^{*}$.}

Let $(X, \leq, \rightarrow)$ be any c.o.s., and let $\left(X^{*}, \varphi\right)$ be the convergence ordered compactification of $X$ constructed in the last section. By Proposition 1.3 there is, for any $f \in C I^{*}(X)$, a unique, continuous, increasing extension $f_{\bullet}: X^{*} \rightarrow[0,1]$.

We define an equivalence relation $R$ on $X^{*}$ as follows: $R=\left\{(\mathcal{F}, \mathcal{G}) \in X^{*} \times X^{*} \quad: \quad f_{*}(\mathcal{F})=\right.$ $f_{*}(\mathcal{G})$, for all $f \in C I^{*}(X)$ \}. Let $\sigma$ be the projection map of $X^{*}$ onto $X^{*} / R$ (i.e., for each $\mathcal{F} \in$ $X^{\bullet}, \sigma(\mathcal{F})=[\mathcal{F}]$, where $[\mathcal{F}]$ is the $R$-equivalence class containing $\mathcal{F}$ ). A partial order $\leq_{R}$ on $X^{\bullet} / R$ is defined as follows:

$$
[\mathcal{F}] \leq_{R}[\mathcal{G}] \text { iff } f_{*}(\mathcal{F}) \leq f_{*}(\mathcal{G}) \text { in } R \text { for all } f \in C I^{*}(X)
$$

We also impose on $X^{*} / R$ the quotient convergence structure which is described (see [2]) as

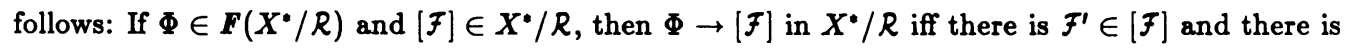
a filter $A \in F\left(X^{*}\right)$ such that $A^{*} \rightarrow F^{\prime}$ in $X^{*}$ and $\sigma(A) \leq \Phi$.

THEOREM 2.1. For any c.o.s. $X, X^{*} / R$ is a compact, $T_{2}$-ordered c.o.s. 
PROOF. $X \cdot / R$ is obviously compact. To show that $X \cdot / R$ is $T_{2}$-ordered, it is sufficient (by Proposition 1.2, [4]) to show that if $\Phi, \Theta \in F\left(X^{*} / R\right), \Phi \rightarrow[\mathcal{F}]$ and $\Theta \rightarrow[\mathcal{G}]$ in $X^{*} / R$, and $\Phi \times \Theta$ has a trace on the order $\leq_{R}$, then $[\mathcal{F}] \leq_{R}[\mathcal{G}]$.

If $f \in C I^{*}(X)$, define $f_{R}: X^{*} / R \rightarrow[0,1]$ by $f_{R}([\xi])=f_{\bullet}(\xi)$, for all $\xi \in X^{\bullet}$. It is easy to verify that $f_{R}$ is well-defined and $f_{R} \in C I^{*}\left(X^{*} / R\right)$. If $\Phi \rightarrow[F]$ and $\Theta \rightarrow[G]$ in $X^{*} / R$ and $\Phi \times \Theta$ has a trace on $\leq_{R}$, it follows that $f_{R}(\Phi) \times f_{R}(\Theta)$ has a trace on the order of $[0,1]$; since $[0,1]$ is $T_{2}$-ordered, $f_{R}([\mathcal{F}])=f_{*}(\mathcal{F}) \leq f_{*}(\mathcal{G})=f_{R}([G])$. The latter inequality holds for all $f \in C I^{*}(X)$, and so $[\mathcal{F}] \leq_{R}[\mathcal{G}]$, which establishes that $X * / R$ is $T_{2}$-ordered.

For an arbitrary c.o.s. $X$, we have already defined the continuous, increasing maps $\varphi: X \rightarrow X^{*}$ and $\sigma: X^{*} \rightarrow X^{*} / R$; we define $\varphi_{R}: X \rightarrow X^{*} / R$ by $\varphi_{R}=\sigma \circ \varphi$. It is clear that $\varphi_{R}(X)$ is dense in the compact, $T_{2}$-ordered c.o.s. $X^{*} / R$. We are now interested in characterizing those spaces $X$ for which $\left(X^{*} / R, \varphi_{R}\right)$ is a compactification. With this goal in mind, we introduce the following conditions.

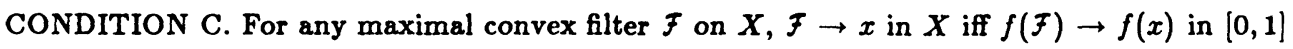
for all $f \in C I^{*}(X)$.

CONDITION $O$. For any points $x, y$ in $X, x \leq y$ in $X$ iff $f(x) \leq f(y)$ in $[0,1]$, for all $f \in C I^{*}(X)$.

It is easy to verify that any $T_{3.5}$-ordered t.o.s. satisfies Conditions $C$ and $O$.

LEMMA 2.2. If $X$ is a c.o.s. satisfying Conditions $C$ and $O$, then $[\dot{x}]=\{\dot{x}\}$, for all $x \in X$.

PROOF. $C I^{*}(X)$ separates points in $X$ by Condition $O$, and so $\sigma$ is one-to-one on $\varphi(X)$. This implies $\dot{y} \notin[\dot{x}]$ if $x \neq y$. Next, assume that there is $\mathcal{F} \in X^{\prime} \cap[\dot{x}]$. Then $f_{*}(\mathcal{F})=f_{*}(\dot{x})=f(x)$ for all $f \in C I^{*}(X)$; in other words, $f(\xi) \rightarrow f(x)$ in $R$, for all $f \in C I^{*}(X)$. Condition $C$ then implies $\mathcal{F} \rightarrow x$ in $X$, contradicting the assumption $\mathcal{F} \in X^{\prime}$.

THEOREM 2.3. Let $X$ be a c.o.s. Then $\varphi_{R}: X \rightarrow X * / R$ is an order and a homeomorphic embedding iff $X$ satisfies Conditions $\mathrm{C}$ and $\mathrm{O}$.

PROOF. Suppose that $X$ satisfies Conditions $\mathrm{C}$ and $\mathrm{O}$. Then $\varphi_{R}$ is one-to-one since $C I^{*}(X)$ separates points in $X$. Also note that $\varphi_{R}=\sigma \cdot \varphi=\left(\left.\sigma\right|_{\varphi(X)}\right) \circ \varphi$, and thus $\left.\sigma\right|_{\varphi(X)}$ is one-to-one.

Let $\Phi \rightarrow[\dot{x}]$ in $X^{*} / R$. Then there is $A \in F\left(X^{*}\right)$ such that $A \stackrel{*}{\rightarrow} \dot{x}$ in $X^{*}$ and $\Phi \geq \sigma(A)$. By definition of * convergence in $X^{*}$, there is a filter $\mathcal{F}$ on $X$ such that $\mathcal{F} \rightarrow x$ and $A \geq \mathcal{F}^{*}$. Therefore, $\varphi_{R}^{-1}(\Phi) \geq \varphi_{R}^{-1}(\sigma(A)) \geq \varphi_{R}^{-1}\left(\sigma\left(F^{*}\right)\right)=\varphi^{-1} \cdot\left(\left.\sigma\right|_{\varphi(X)}\right)^{-1}\left(\sigma\left(F^{*}\right)\right)$. It follows by Lemma 2.2 that $\left(\left.\sigma\right|_{\varphi(X)}\right)^{-1}\left(\sigma\left(\mathcal{F}^{*}\right)\right) \geq \mathcal{F}^{*}$. Consequently, $\varphi_{R}^{-1}(\Phi) \geq \varphi^{-1}\left(\mathcal{F}^{*}\right)=\mathcal{F} \rightarrow x=\varphi_{R}^{-1}([\dot{x}])$, i.e. $\varphi_{R}^{-1}(\Phi) \rightarrow \varphi_{R}^{-1}([\dot{x}])$. Thus $\varphi_{R}^{-1}$ is continuous.

Let $[\dot{x}] \leq_{R}[\dot{y}]$ in $X^{*} / R$; then for any $f \in C I^{*}(X), f_{*}(\dot{x}) \leq f_{*}(\dot{y})$, i.e. $f_{*}(\varphi(x)) \leq f_{*}(\varphi(y))$, which implies $f(x) \leq f(y)$, for all $f \in C I^{*}(\mathbf{X})$. By Condition $O, x \leq y$. Thus $\varphi_{R}^{-1}$ is increasing, and we conclude that $\varphi_{R}$ is an order and homeomorphic embedding.

Conversely, assume that $\varphi_{R}$ is both an order and homeomorphic embedding. Let $\mathcal{F}$ be a maximal convex filter on $X$ such that, for some $x \in X, f(\mathcal{F}) \rightarrow f(x)$ for all $f \in C I^{*}(X)$. Suppose $\mathcal{F} \rightarrow x$ is not true. Then we need to consider two cases.

CASE 1. $F \rightarrow y$ and $y \neq x$. This implies that for each $f \in C I^{*}(X), f(\mathcal{F}) \rightarrow f(y)$. From this we deduce that $[\dot{x}]=[\dot{y}]$, which is a contradiction, since $\varphi_{R}$ is assumed to be one-to-one.

CASE 2. $\mathcal{F} \in X^{\prime}$. This leads to the conclusion that $[\mathcal{F}]=[\dot{x}]$; in other words, $\varphi_{R}(\mathcal{F}) \rightarrow[\dot{x}]$ in $X^{*} / R$, which implies $\mathcal{F} \rightarrow x$ in $X$, since $\varphi_{R}$ is a homeomorphic embedding. This contradicts $\mathcal{F} \in X^{\prime}$. We therefore conclude that $X$ satisfies Condition $C$.

Finally, let $x, y \in X$ such that $f(x) \leq f(y)$ for all $f \in C I^{*}(X)$. Then $f_{\bullet}(\varphi(x)) \leq f_{\bullet}(\varphi(y))$ for all $f \in C I^{*}(X)$, i.e. $f_{*}(\dot{x}) \leq f_{*}(\dot{y})$ for all $f \in C I^{*}(X)$. This implies $[\dot{x}] \leq_{R}[\dot{y}]$ in $X^{*} / R$, and $x \leq y$ 
follows since $\varphi_{R}$ is an order embedding. Therefore, $X$ satisfies Condition $O$.

THEOREM 2.4. For every c.o.s. $X$ satisfying Conditions $C$ and $O,\left(\left(X^{*} / R\right), \varphi_{R}\right)$ is a $T_{2}$-ordered c.o.s. compactification of $X$. Furthermore, for any compact, regular, $T_{2}$-ordered c.o.s. $Y$ and for any continuous, increasing map $f: X \rightarrow Y$, there is a unique, continuous, increasing extension $f_{R}: X * / R \rightarrow Y$.

PROOF. The first assertion is an immediate corollary of Theorem 2.3. The second follows easily with the help of Proposition 1.3.

For any c.o.s. $X$, let $\omega_{0} X$ be the t.o.s. consisting of the poset $(X, \leq)$ with the weak topology induced by $C I^{*}(X)$. Note that $C I^{*}(X)=C I^{*}\left(\omega_{0} X\right)$.

PROPOSITION 2.5. Let $X$ be a c.o.s. satisfying Condition $C$. Let $i: X \rightarrow \omega_{0} X$ be the identity map. Then $i$ is an order isomorphism and a homeomorphism relative to ultrafilter convergence.

PROOF. It is obvious that $i$ is a continuous order isomorphism. Let $F \rightarrow x$ in $\omega_{0} X$, where $\mathcal{F}$ is an ultrafilter. By Proposition 1.2, $\hat{f}$ is a maximal convex filter and $f(\mathcal{F}) \rightarrow f(x)$ implies $f(\hat{f}) \rightarrow f(x)$ in $[0,1]$, for all $f \in C I^{*}(X)$. Condition $C$ thus guarantees that $\hat{f} \rightarrow x$ in $X$, and hence $\mathcal{F} \rightarrow x$ in $X$.

PROPOSITION 2.6. If $X$ is a c.o.s. satisfying Conditions $C$ and $O$, then $\omega_{0} X$ is a $T_{3.5}$-ordered t,o.s.

PROOF. First observe that $\omega_{o} X$ also satisfies Condition $C$ and $O ; O$ is obvious, and $C$ follows from Proposition 2.5, since $X$ and $\omega_{0} X$ have the same ultrafilter convergence and hence, by Proposition 1.2, the same convergence of maximal convex filters.

For $f \in C I^{*}\left(\omega_{0} X\right)$, let $I$ be the closed interval $[0,1]$ indexed by $f$, and let $P=\Pi\left\{I_{f}: f \in\right.$ $\left.C I^{*}(X)\right\}$ be equipped with the usual product order and product topology. Then $P$ is a compact, $T_{2}$-ordered t.o.s. Define $\varphi_{0}: \omega_{0} X \rightarrow P$ by $\varphi_{0}(x)=\hat{x}$, where $\hat{x}: C I^{*}\left(\omega_{0} X\right) \rightarrow[0,1]$ is given by $\hat{x}(f)=f(x)$, for all $f \in C I^{*}\left(\omega_{0} X\right)$. Since $\omega_{0} X$ has the weak topolegy induced by $C I^{*}\left(\omega_{0} X\right)=$ $C I^{*}(X)$, and $C I^{*}\left(\omega_{0} X\right)$ separates points in $\omega_{0} X$ by Condition $O, \varphi_{0}$ is a topological embedding (see 8.12, [10]). By Condition $O, \varphi_{0}$ is also an order embedding.

Given a c.o.s. $X$ satisfying $C$ and $O$, we introduce some additional functional notation. Let $e_{0}$ be the evaluation embedding of the $T_{3.5}$-ordered t.o.s. $\omega_{0} X$ into its Nachbin compactification $\beta_{0} X$, and let $e=e_{0} \cdot i: X \rightarrow \beta_{0}\left(\omega_{0} X\right)$. The unique extension of $e$ to $X^{*}$ (guaranteed by Proposition 1.3) is denoted by $e_{*}$, and the extension of $e$ to $X^{*} / R$ (guaranteed by Theorem 2.4) is denoted by $e_{R}$. If $f \in C I^{*}(X)=C I^{*}\left(\omega_{0} X\right)$, the unique extensions of $f$ in $C I^{*}\left(X^{*}\right)$ and $C I^{*}\left(\beta_{o}\left(\omega_{0} X\right)\right)$ (see Proposition 1.3 and 2.4) are denoted by $f_{*}$ and $f^{*}$, respectively. The following commutative diagram is helpful in keeping track of these various maps.

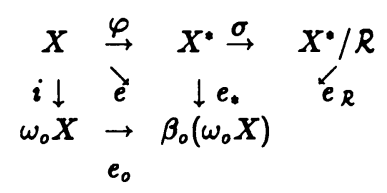

THEOREM 2.7. If $X$ is any c.o.s. satisfying $C$ and $O$, then $e_{R}$ is an order isomorphism and a homeomorphism relative to ultrafilter convergence.

PROOF. Since $[\mathcal{F}]=[\mathcal{G}]$ in $X^{*} / R$ iff $e_{*}(\mathcal{F})=e_{*}(\mathcal{G})$ iff $e_{R}([\mathcal{F}])=e_{R}([\mathcal{G}])$, it follows that $e_{R}$ is one-to-one. Furthermore, $e(X)$ is dense in $\beta_{0}\left(\omega_{0} X\right)$, which implies that the extension $e_{\mathcal{R}}$ is onto $\beta_{0}\left(\omega_{0} X\right)$. It follows from Theorem 2.4 that $e_{R}$ is continuous and increasing. Finally, if $\mathcal{K}$ is an ultrafilter on $\beta_{o}\left(\omega_{0} X\right)$ and $\mathcal{H} \rightarrow a$ in $\beta_{o}\left(\omega_{0} X\right)$, then there is $\alpha \in X^{*} / R$ such that $e_{R}^{-1}(\mathcal{H}) \rightarrow \alpha$ in 
$X * / R$ since the latter space is compact. It follows by uniqueness of filter limits in both spaces and the continuity of $e_{R}$ that $e_{R}^{-1}(a)=\alpha$.

If $X$ is any convergence space, let $\lambda X$ denote its topological modification (i.e., $\lambda X$ is the set $|X|$ equipped with the finest topological structure coarser than $X$.) If $X$ is a c.o.s. satisfying $C$ and $O$, we obtain from Proposition 2.5 and Theorem 2.7 that $\lambda X=\omega_{0} X$ and $\lambda\left(X^{*} / R\right)$ is a compact, $T_{2}$-ordered t.o.s. homeomorphic and order isomorphic under $e_{R}$ to $\beta_{0}\left(\omega_{0} X\right)$. Let $\varphi_{0}: \omega_{0} X \rightarrow X * / R$ be defined by $\varphi_{0}=\sigma \circ \varphi \circ i^{-1}=\varphi_{R} \circ i^{-1}$.

COROLLARY 2.8. If $X$ is a c.o.s. satisfying $C$ and $O$, then $\left(\lambda\left(X^{*} / R\right), \varphi_{0}\right)$ is the Nachbin compactification of $\omega_{0} X=\lambda X$. If $X$ is a $T_{3.5}$-ordered t.o.s., then $\left(\lambda(X \cdot / R), \varphi_{0}\right)$ is the Nachbin compactification of $X$.

One question which deserves clarification is the status of $X^{\bullet} / R$ as a "quotient" of $X^{\bullet}$. We have indeed equipped $X \cdot / R$ with the quotient convergence structure, but can we interpret $\leq_{R}$ as the "quotient order" relative to the order $\leq$ " defined on $X *$ ? Various notions of "quotient order" have been considered (for instance, see [5] and [8]), but the order $\leq_{R}$ is generally different than these. Instead of regarding the order and convergence structures of $X^{*} / R$ separately, we think that it is appropriate to consider the notion of a "quotient c.o.s.", where order and convergence structures are considered together. From this perspective, the next theorem indicates that $X * / R$ is indeed a quotient c.o.s. of $X^{*}$, at least in the category of c.o.s.'s which satisfy Conditions $C$ and $O$.

THEOREM 2.10. For a c.o.s. $X$, let $X^{*}$ and $X^{*} / R$ be defined as before. Let $Y$ be any c.o.s. satisfying $C$ and $O$, and let $h: X^{*} / R \rightarrow Y$. Then $h$ is continuous and increasing iff $h \circ \sigma: X^{\bullet} \rightarrow Y$ is continuous and increasing.

PROOF. If $h$ is continuous and increasing, the same is obviously true for $h \circ \sigma$.

Conversely, suppose $h \circ \sigma$ is continuous and increasing. Let $\Phi \rightarrow[\exists]$ in $X^{*} / R$; then there is $\mathcal{F}^{\prime} \in[\mathcal{F}]$ and a filter $A$ on $X^{*}$ such that $A \rightarrow \mathcal{F}^{\prime}$ in $X^{*}$ and $\Phi \geq \sigma(A)$. Hence $h \circ \sigma(A) \rightarrow h \circ \sigma\left(F^{\prime}\right)$ in $Y$, by continuity of $h \circ \sigma$. But $\Phi \geq \sigma(A)$ and $\sigma\left(\mathcal{F}^{\prime}\right)=[\mathcal{F}]$, so $h(\Phi) \rightarrow h([\mathcal{F}])$, implying that $h$ is continuous.

To show that $h$ is increasing, let $e_{Y}$ be the natural map from $Y$ into $\beta_{0}\left(\omega_{0} Y\right)$ and consider $g=e_{Y} \circ h \circ \sigma \circ \varphi: X \rightarrow \beta_{0}\left(\omega_{0} Y\right)$. Since $g: \omega_{0} X \rightarrow \beta_{0}\left(\omega_{0} Y\right)$ is also continuous and increasing, there is a continuous, increasing extension $g^{*}: \beta_{0}\left(\omega_{0} X\right) \rightarrow \beta_{0}\left(\omega_{0} Y\right)$ which makes the diagram below commute.

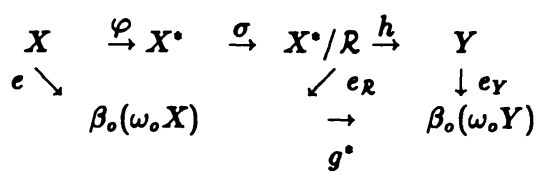

Thus $e_{Y} \circ h \circ \sigma \circ \varphi=g^{*} \circ e_{R} \circ \sigma \circ \varphi$, and since $\sigma \circ \varphi: X \rightarrow X \cdot / R$ is a dense injection, $e_{Y} \circ h=g^{*} \circ e_{R}$. But $e_{Y}$ is an order embedding, so $h=e_{Y}^{-1} \circ g^{*} \circ e_{R}$, and $h$ is increasing.

\section{3. $T_{3.5}$-ORDERED CONVERGENCE ORDERED SPACES.}

In this brief concluding section, we introduce the notion of a $T_{3.5}$-ordered c.o.s., describe the largest regular, $T_{2}$-ordered c.o.s. compactification of such a space, and interpret this compactification in the language of category theory. The necessary categorical terminology can be found in [7].

In [3], a convergence space $X$ is defined to be completely regular if it allows a symmetric com- 
pactification. In [9], it is shown that the Hausdorff, completely regular convergence spaces, which we shall refer to as $T_{3.5}$ convergence spaces, are precisely those convergence spaces which allow a regular, Hausdorff convergence space compactification.

Given a convergence space $X$, let $r X$ denote the regular modification of $X$ (i.e., $r X$ is the set $|X|$ equipped with the finest regular convergence structure coarser than the original convergence structure on $X$ ).

We define a c.o.s. $X$ which is regular and satisfies conditions $C$ and $O$ to be a $T_{3.5}$-ordered c.o.s.. It follows by Proposition 2.5 that a $T_{3.5}$-ordered c.o.s. $X$ has the same ultrafilter convergence as its topological modification $\lambda X=\omega_{0} X$.

THEOREM 3.1. Let $X$ be a $T_{3.5}$-ordered c.o.s. and let $\eta_{0} X=r\left(X^{*} / R\right)$ be the regular modification of $X^{*} / R$. Then $\left(\eta_{0} X, \varphi_{R}\right)$ is a regular, $T_{2}$-ordered c.o.s. compactification of $X$. If $Y$ is a regular, $T_{2}$-ordered, compact c.o.s. and $f: X \rightarrow Y$ is continuous and increasing, then $f$ has a unique, continuous, increasing extension $f_{\bullet}: \eta_{0} X \rightarrow Y$.

PROOF. By Theorem 2.3, $\varphi_{R}: X \rightarrow X * / R$ is an order embedding and a homeomorphic embedding. By the functorial properties of the regular modification and the fact that $r X=X$, it follows that $\varphi_{R}: X \rightarrow \eta_{0} X$ is continuous. Because $X * / R$ and $\eta_{0} X$ have the same ultrafilter convergence, it is easy to verify that the regular modification of $\varphi_{R}(X)$ (considered as a subspace of $\left.X^{*} / R\right)$ coincides with $\varphi_{R}(X)$ considered as a subspace of $\eta_{0} X$. From this we see that $\varphi_{R}^{-1}$ is also continuous, and the first assertion is established. The second assertion is an immediate consequence of Theorem 2.4.

We denote by $C$ the category of all $T_{3.5}$ ordered c.o.s.'s, with increasing continuous maps as morphisms; let $D$ be the full subcategory of $C$ consisting of all regular, compact, $T_{2}$-ordered c.o.s.'s. If $\iota: D \rightarrow C$ is the inclusion functor, it follows by Theorem 3.1 that the functor $\eta_{0}: C \rightarrow D$, which assigns to each object $X$ in $C$ its compactification $\eta_{0} X$ and to each morphism $f: X \rightarrow Y$ in $C$ the extension $f_{*}: \eta_{0} X \rightarrow \eta_{0} Y$ whose existence follows by Theorem 3.1, is the left adjoint of $\iota$.

THEOREM 3.2. If $\boldsymbol{C}$ and $\boldsymbol{D}$ are the categories defined in the preceding paragraph, then $\boldsymbol{D}$ is an epireflective subcategory of $C$.

If $X$ is a $T_{3.5}$-ordered t.o.s., it is generally not true that $\beta_{0} X=\eta_{0} X$, although it is true in this case that $\beta_{0} X=\lambda\left(\eta_{0} X\right)$.

The $T_{3.5}$ convergence spaces mentioned earlier in this section are the $T_{3.5}$-ordered c.o.s.'s for which the partial order is equality. Indeed, any $T_{3.5}$ convergence space $X$, equipped with the trivial order (equality), satisfies Condition $C$ and $O$ relative to $C I^{*}(X)=C^{*}(X)$, the set of all continuous maps from $X$ into $[0,1]$. For such a space $X, \eta_{0} X$ (which also has the trivial order) coincides with the largest regular, Hausdorff convergence space compactification of $X$ constructed in [9].

\section{REFERENCES}

[1] P. Fletcher and W. Lindgren, Quasi-Uniform Spaces, Lect. Notes in Pure and Appl. Math., Vol. 77, Marcel Dekker, Inc., New York (1982).

[2] D.C. Kent, "Convergence Quotient Maps", Fund. Math. 65 (1969) 197-205.

[3] D.C. Kent and G.D. Richardson," Completely Regular and $\omega$-Regular Spaces", Proc. Amer. Math. Soc. 82 (1981), 649-652.

[4] , “A Compactification for Convergence Ordered Spaces", Canad. Math. Bull. 27 (1984) 505-512. 
[5] S.D. McCartan, “A Quotient Ordered Space”, Proc. Camb. Phil. Soc. 64 (1968), 317-322.

[6] L. Nachbin, Topology and Order, Van Nostrand, New York Math. Studies, 4, Princeton, N.J. (1965).

[7] G. Preuss, Theory of Topological Structures, D. Reidel Publ. Co., Dordrecht (1987).

[8] H.A. Priestley, "Ordered Topological Spaces and the Representation of Distributive Lattices", Proc. London Math. Soc. (S) 24 (1972), 507-530.

[9] G.D. Richardson and D.C. Kent, "Regular Compactification of Convergence Spaces", Proc. Amer. Math. Soc. 31 (1972), 571-573.

[10] S. Willard, General Topology, Addison-Wesley Publ. Co., Reading, Mass. (1970). 


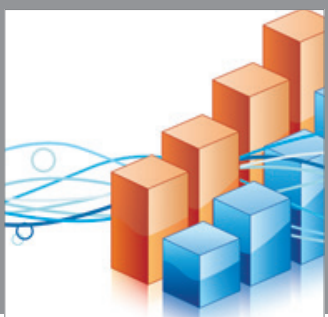

Advances in

Operations Research

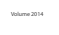

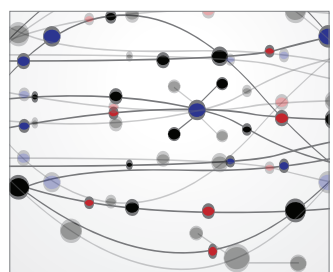

\section{The Scientific} World Journal
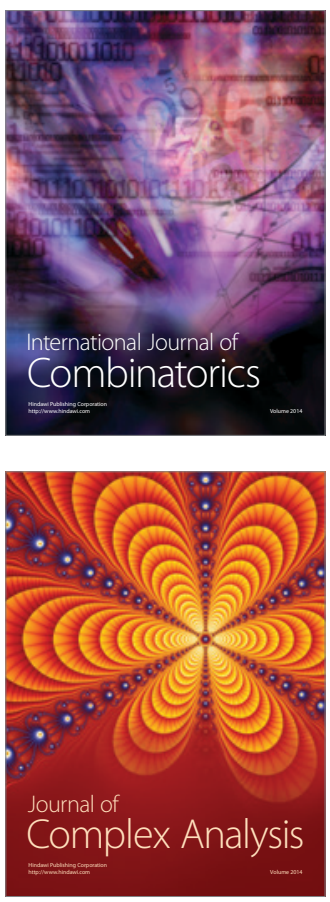

International Journal of

Mathematics and

Mathematical

Sciences
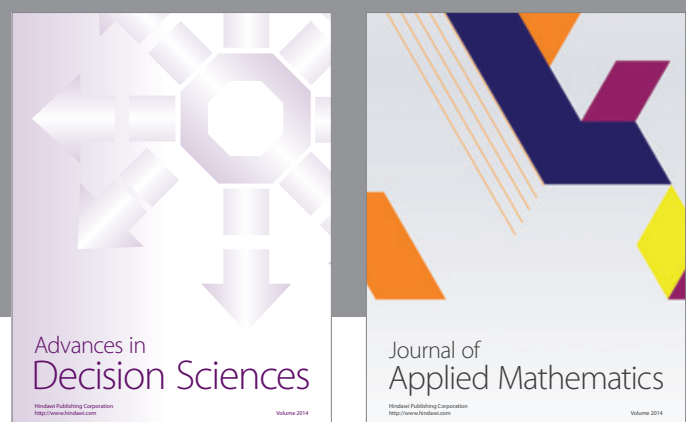

Journal of

Applied Mathematics
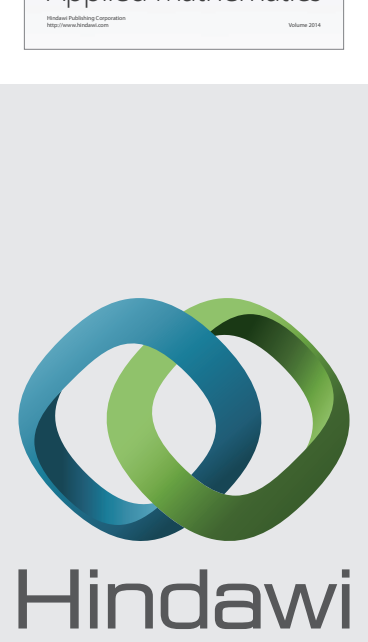

Submit your manuscripts at http://www.hindawi.com
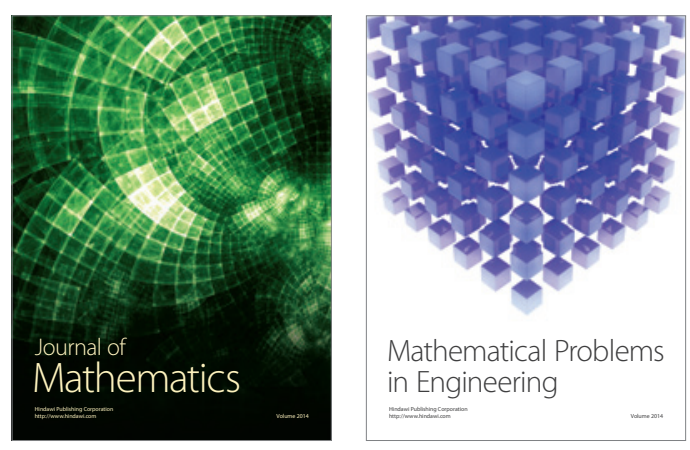

Mathematical Problems in Engineering
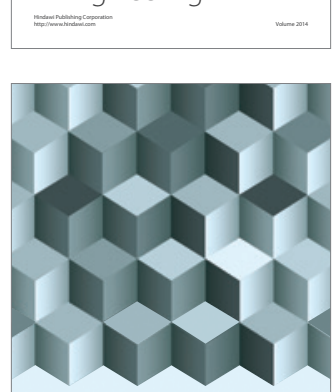

Journal of

Function Spaces
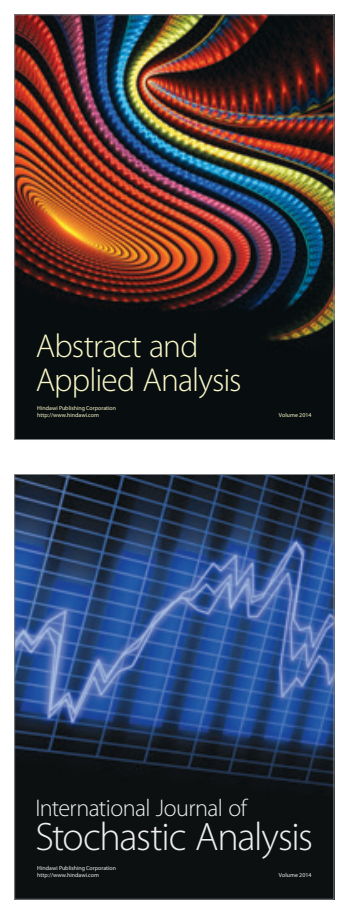

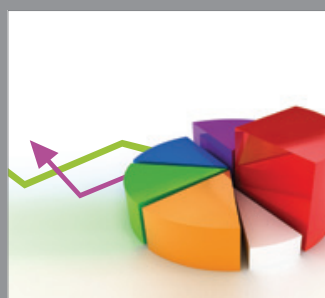

ournal of

Probability and Statistics

Promensencen
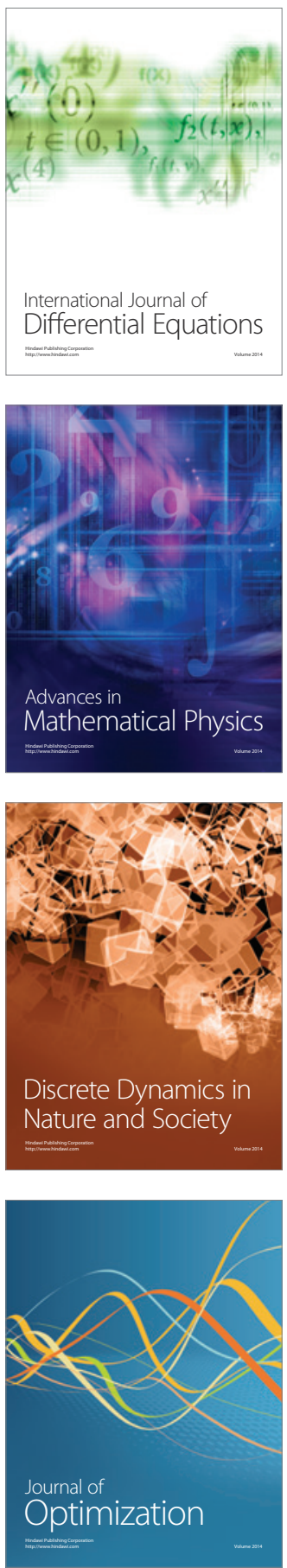